\title{
Discovering significant patterns
}

\section{Geoffrey I. Webb}

Published online: 2 February 2008

Springer Science+Business Media, LLC 2008

\section{Erratum to: Mach Learn (2007) 68:1-33 \\ DOI: 10.1007/s10994-007-5006-x}

The following error appeared in the paper by G. I. Webb, "Discovering significant patterns," published in volume 68 number 1.

On page 5, the sentence "Redundant rule constraints discard rules $x \rightarrow y$ for which $\exists z \in x: \operatorname{support}(x \rightarrow y)=\operatorname{support}(x-z \rightarrow y)$ " should read "Redundant rule constraints discard rules $x \rightarrow y$ for which $\exists z \in x:$ coverage $(x \rightarrow y)=\operatorname{coverage}(x-z \rightarrow y)$."

The online version of the original article can be found at http://dx.doi.org/10.1007/s10994-007-5006-x. 